\title{
Functional prognosis in stroke: use of somatosensory evoked potentials
}

\author{
B D ZEMAN, C YIANNIKAS
}

From the Department of Neurophysiology, Westmead Hospital, Westmead, NSW, Australia

SUMMARY Median nerve somatosensory evoked potentials (SEPs) were performed on 35 patients $\overrightarrow{0}$ with acute stroke and correlated with functional outcome as measured by placement, length of stay $\frac{\bar{\sigma}}{\bar{\rho}}$ and an activities of daily living index (Barthel Index). There was a statistically significant correlation $\frac{\hat{\sigma}}{\Phi}$ of both SEP and sensory examination at the 0.05 level to eventual functional outcome. SEPs were $\varrho$ better than age, sex and side of CVA in predicting functional outcome as measured by these scores. SEPs and sensory examination are interrelated but SEPs offer the advantages of objectivity, the $\vec{\circ}$ ability to quantify results and the use in unconscious or dysphasic patients.

The ability to predict functional outcome in stroke patients has great importance in developing effective forms of therapy, in resource allocation and in individual consequences. Although functional outcome can be predicted to a certain extent with clinical examination, age, sex, side and site of stroke,,$^{1-4}$ these factors are not sensitive enough and are only suitable for large groups of patients.

There are no universally accepted measures of functional outcome, so a number of indirect measures can be used. Placement (that is, return home or to a nursing home) and length of hospital stay are frequently used as indirect measures of functional outcome. Many indices of activities of daily living (ADL) have been developed to give approximations for selected activities.

The Barthel Index ${ }^{5}$ is a well recognised and accepted index of ADL. It scores functional outcome out of a possible score of 100 . Being independent although possibly still having neurological deficits is scored 100 .

Since Dawson in $1947^{\circ}$ described the technique of somatosensory evoked potentials (SEPs), there have been many publications about their use in various neurological conditions. These include multiple sclerosis, head injuries, brachial plexus injuries, spinal injuries etc. ${ }^{7-9}$ Short latency somatosensory evoked potentials are those with a latency of less than $100 \mathrm{~ms}$. They most accurately reflect proprioceptive pathways and their direct synaptic connections including posterior and posterolateral columns.

Although there have been studies on the use of SEPs

Address for reprint requests: Dr Brian Zeman, Coorabel Hospital, 227 Morrison Rd, Ryde, 2112, Australia.

Received 22 January 1988 and in revised form 24 September 1988. Accepted 27 September 1988 in stroke, most have concentrated on the correlation with the neurological examination, few have looked at 0 function and none have used an accepted index of $\mathrm{N}$ function and ADL. In addition, there have been $\mathrm{N}$ various design limitations. Some studies have looked is at only small numbers of patients, ${ }^{10-12}$ and others hat included a range of pathological conditions. ${ }^{13} \stackrel{\phi}{\Phi}$ 음 Frequently, there were large time variables betwe stroke and SEP testing.

It has been established that multimodality evokeg 을 potentials can predict outcome in head injuries ${ }^{16} 17$ and more recently Cant et $a{ }^{18}$ proposed that SEPs alowe can predict short term outcome in head injuries. The $\varphi_{\infty}$ is some suggestive evidence that they may be a useful. prognostic indicator in stroke..$^{10-1219}$ It was felt therefore that SEPs might be useful in predicting functional outcome in stroke.

The aim of this study was specifically to look at the usefulness of SEPs in predicting outcome as measured $\stackrel{\mathcal{Q}}{\mathcal{Q}}$ by placement, length of stay and Barthel Index. In $\overrightarrow{\vec{F}}$ addition, its practical application is compared with $\frac{3}{3}$ current other methods of prognostication.

\section{Materials and methods}

\section{(a) Patients}

The patient population consisted of acute stroke patients referred to the rehabilitation period from May 1985 to May. 1986 and also included a small number of patients who had $\bar{\Omega}$ been used as a pilot programme over a 10 week period in 1984. The criteria for referral to the rehabilitation unit were 0 all acute strokes admitted to Westmead Hospital under the age of 72 years with a neurological deficit. Patients with $\frac{7}{0}$ TIAs, multiple infarcts and peripheral neuropathies were excluded. There were no exclusions for the study on the basis $N$ of rapid recovery or very poor outcome. Four patients were excluded because of peripheral neuropathy as evidenced by abnormal brachial plexus potentials. In the remaining $35 \mathrm{~W}$ 
patients the mean age was 56.4 years; the range 15 to 72 yrs and the median age was 62 years. (There were 18 men and 17 women). All patients who were given a rehabilitation programme, had this by therapists who were not aware of the SEP result.

Patients had the test explained and permission was given before testing. All patients had CT examinations. Twenty nine had CT evidence of infarction; six patients had normal CT scans (all of these were repeated and still showed no abnormality). There were two patients with brainstem infarcts (one haemorrhagic), 11 patients had subcortical infarcts (basal ganglia, corona radiata or internal capsule) and 16 patients with cortical infarcts. Fifteen of these were in the distribution of the middle cerebral artery and one in the distribution of the posterior cerebral artery.

\section{(b) Controls}

SEPs were performed on 26 normal controls (age range 19-83 years); mean age 50-65 years. There were 14 women and 12 men.

\section{(c) SEP method}

SEPs were performed using a similar technique to that previously described by Chiappa. ${ }^{8}$ Recording was performed using surface silver chloride electrodes overlying Erb's point, the upper cervical vertebrae (C2) and the contralateral cortex overlying the primary somatosensory area (CC) with a common mid frontal reference electrode ( $\mathrm{Fz}$ : 10-20 system). An electrode was also placed over the ipsilateral cortex. All four channels were recorded simultaneously. In selected patients an additional non cephalic reference site was used.

The stimulus was a square wave of $100 \mu$ s duration delivered to the skin with the cathode overlying the median nerve at the wrist and proximal to the anode. The stimulus strength was adjusted to produce a twitch of the appropriate muscles at a frequency of $2 \mathrm{~Hz}$. Subjects sat in an armchair and were instructed to relax and to sleep if they wished. The recording electrodes were connected to a Digitimer D200, 256 stimulations were performed and repeated for reproducibility. The bandpass used was $16 \mathrm{~Hz}$ to $3 \mathrm{KHz}$. Each sweep was from $0 \mathrm{~ms}$ to $52 \mathrm{~ms}$. The digital averaging system sampled 256 points per ms. The procedure was performed on both arms for comparison purposes. Final results were plotted on paper with a Hewlett Packard 7470A plotter.

Lower limb evoked potentials were performed on some patients as it was intended to correlate these with outcome but technical difficulties in performing this and in particular obtaining the lumbar potential to determine latencies meant that only a small number of patients could be examined and so this was abandoned for the study.

Latency and amplitude were measured of the first negative peak over Erb's point (N9), cervical spine (N13) and contralateral cortex (N20). Interpeak latencies were calculated from these points. In addition, amplitude of the positivity following $\mathrm{N} 20(\mathrm{P} 23 / \mathrm{P} 30)$ was compared to the opposite side.

An abnormal SEP was judged as having any of the following: An absent N20; an amplitude of N20 less than $50 \%$ of the unaffected cortex or less than the lowest value in the normal range; a prolonged latency of $\mathrm{N} 20$ (more than 2.5 times the standard deviation of controls); a reduction in amplitude of the following positivity of greater than $50 \%$ of the unaffected side.

\section{(d) Functional outcome}

Decisions for discharge, placement and scoring of Barthel Index were done independently of the treating clinicians. Decisions for discharge were determined uniformly by whether the patient could manage at home and not for other reasons such as pressure from hospital bed shortages.

The patients were all examined by a neurologist and a rehabilitation specialist and a standard neurological examination was performed at the time of testing and at follow up. Follow up ceased when patients had plateaued neurologically and functionally. A score of 100 meant they were able to perform these selected activities but that they still may have neurological impairment.

\section{(e) Statistics}

Statistical analysis was performed to determine the level of significance. " $z$ " values were derived using the formula:

$$
z=\frac{(x-a)}{b} \text { multiplied by the square root of } n .
$$

where $x$ is the mean of the sample size $n ; a$ is the mean of the total group and $b$ is the standard deviation of the total group. The level of significance was then derived from tables using a one-tailed normal curve.

\section{Results}

One patient died from bacterial endocarditis after open heart surgery prior to his stroke and was excluded from length of stay and placement but was included in Barthel Index because he had plateaued.

All other patients went home except for three who were placed in a nursing home. Two others went home against advice and were expected to be placed in a nursing home eventually. Four of these five patients with very poor outcome had abnormal SEPs and severe sensory loss. The small numbers involved preclude using place of discharge as a useful measure of outcome but these results indicate that those with an abnormal SEP are more likely to require placement in a nursing home.

There were 19 patients with abnormal SEPs (henceforth referred to as Group I) and 16 patients with normal SEPs (henceforth referred to as Group II). They were comparable by age and sex. SEPs were performed between 4 and 34 days after the stroke (average $12 \cdot 14$ days). Those patients with an abnormal SEP result had the test performed 14.63 days after their stroke compared to $9 \cdot 18$ days for those with normal SEP results. This is insufficient time for there to be any differences in SEP result due to retrograde degeneration.

Results are summarised in tables 1, 2. Normal SEP correlates highly with a good functional outcome as measured by length of stay and Barthel Index. An abnormal SEP correlates with a longer length of stay 
Table 1 Length of stay in days

\begin{tabular}{|c|c|c|c|c|c|}
\hline Group & Average & No & $S D$ & $z$ & $\begin{array}{l}\text { Level of } \\
\text { significance }\end{array}$ \\
\hline $\begin{array}{l}\text { Total } \\
\text { I (Abnormal SEP) } \\
\text { II (Normal SEP) } \\
\text { Group A \& B (Sensory loss) } \\
\text { Group C (Normal Sensation) } \\
\text { Male } \\
\text { Female } \\
\text { Left CVA } \\
\text { Right CVA } \\
62 \text { yrs and over } \\
\text { Under } 62 \text { yr } \\
\text { Both Abnormal SEP \& Sensory loss } \\
\text { Both Normal SEP \& Normal sensation }\end{array}$ & $\begin{array}{l}51 \cdot 14 \\
70 \cdot 30 \\
29 \cdot 56 \\
70 \cdot 93 \\
33 \cdot 35 \\
52 \cdot 23 \\
50 \cdot 00 \\
48 \cdot 23 \\
65 \cdot 46 \\
60 \cdot 18 \\
43 \cdot 11 \\
83 \cdot 46 \\
32 \cdot 5\end{array}$ & $\begin{array}{l}34 \\
18 \\
16 \\
16 \\
18 \\
17 \\
17 \\
13 \\
15 \\
16 \\
18 \\
13 \\
13\end{array}$ & $\begin{array}{l}42 \cdot 61 \\
38 \cdot 86 \\
36 \cdot 66 \\
42 \cdot 80 \\
34 \cdot 77 \\
47 \cdot 54 \\
38 \cdot 50 \\
43 \cdot 67 \\
44 \cdot 45 \\
45 \cdot 67 \\
39 \cdot 33 \\
37 \cdot 11 \\
38 \cdot 6\end{array}$ & $\begin{array}{l}1.79 \\
1.82 \\
1.85 \\
1.77 \\
0.10 \\
0.11 \\
0.24 \\
1.27 \\
0.84 \\
0.79 \\
2.72 \\
1.57\end{array}$ & $\begin{array}{l}0.037^{*} \\
0.034^{*} \\
0.032^{*} \\
0.038^{*} \\
0.460 \\
0.460 \\
0.405 \\
0.102 \\
0.200 \\
0.215 \\
0.003^{*} \\
0.058\end{array}$ \\
\hline
\end{tabular}

*Indicates a level of significance below $0 \cdot 05$.

and a lower Barthel Index but with a much wider variation. The results also show that SEP abnormality is a better predictor of outcome than age, sex, and side of stroke but that sensory impairment correlates with outcome as well as SEP. Examples of SEP abnormalities are illustrated in figs 1 and 2.

There were 16 patients with sensory impairment; nine with severe sensory loss involving light touch, stereognosis and pinprick (henceforth referred to as Group A) and seven patients with mild to moderate sensory impairment with decreased but not absent either light touch, stereognosis and pinprick (henceforth referred to as Group B). The sensory loss was assessed on both upper and lower limbs and graded on a scale using normal, mild, moderate and severe gradings. It did not differ between upper and lower limbs in the group of patients studied. There were 19 patients with normal sensation (Group C). There were 13 patients with both abnormal SEP and sensory impairment ( $68 \%$ of abnormal SEPs had sensory loss). There were 13 patients who had both a normal SEP and normal sensation $(81 \%$ of normal SEPs had normal sensation). A closer association between SEPs and specific sensory pathways could not be determined in these groups as those with sensory loss usually had all modalities affected to various degrees.

Preliminary findings suggest that the relationship of SEP abnormality to the outcome measures may be dependent on the site of the infarct; an absent SEP response in a cortical infarction having a much poorer outcome than a subcortical lesion. However a larger study is required to confirm the relationship of the SEP, outcome and the site of the stroke. The size of the stroke in cortical infarcts will also have an effect on the outcome and perhaps the SEP reflects this, but much larger number of patients with cortical infarcts would be required to draw any statistically significant cons clusions.

There were 11 patients with a low amplitude response (either N20, P30 or both) and/or a delaye N20. Of these, five had cortical infarcts and five were subcortical (basal ganglia). One patient had a normal CT scan. The outcome in nine of the 11 was good (mean Barthel of 90.5). In two patients the outcome was poor (mean Barthel of 32.5) and both these patients had frontoparietal infarcts. This could

Table 2 Barthel index

\begin{tabular}{|c|c|c|c|c|c|}
\hline Group & Average & No & $S D$ & $z$ & $\begin{array}{l}\text { Level of } \\
\text { significance }\end{array}$ \\
\hline $\begin{array}{l}\text { Total } \\
\text { I (Abnormal SEP) } \\
\text { II (Normal SEP) } \\
\text { Group A \& B (Sensory loss) } \\
\text { Group C (Normal Sensation) } \\
\text { Male } \\
\text { Female } \\
\text { Left CVA } \\
\text { Right CVA } \\
62 \text { yrs and over } \\
\text { Under } 62 \text { yr } \\
\text { Both Abnormal SEP \& Sensory loss } \\
\text { Both Normal SEP \& Normal sensation }\end{array}$ & $\begin{array}{l}85 \cdot 57 \\
76 \cdot 84 \\
95 \cdot 93 \\
74 \cdot 37 \\
95 \cdot 00 \\
82 \cdot 7 \\
88 \cdot 52 \\
81 \cdot 92 \\
82 \cdot 66 \\
80 \cdot 29 \\
90 \cdot 55 \\
68 \cdot 46 \\
95 \cdot 00\end{array}$ & $\begin{array}{l}35 \\
19 \\
16 \\
16 \\
19 \\
13 \\
17 \\
13 \\
15 \\
17 \\
18 \\
13 \\
13\end{array}$ & $\begin{array}{l}22 \cdot 58 \\
24 \cdot 50 \\
14 \cdot 96 \\
24 \cdot 13 \\
13 \cdot 74 \\
24 \cdot 92 \\
20 \cdot 13 \\
30 \cdot 03 \\
18 \cdot 79 \\
26 \cdot 24 \\
17 \cdot 81 \\
25 \cdot 52 \\
16 \cdot 58\end{array}$ & $\begin{array}{l}1.65 \\
1.82 \\
1.97 \\
1.62 \\
0.52 \\
0.53 \\
0.57 \\
0.48 \\
0.95 \\
0.92 \\
2.71 \\
1.49\end{array}$ & $\begin{array}{l}0.049^{*} \\
0.034^{*} \\
0.024^{*} \\
0.053 \\
0.300 \\
0.298 \\
0.284 \\
0.316 \\
0.171 \\
0.179 \\
0.003^{*} \\
0.068\end{array}$ \\
\hline
\end{tabular}

*Indicates a level of significance below 0.05 . 

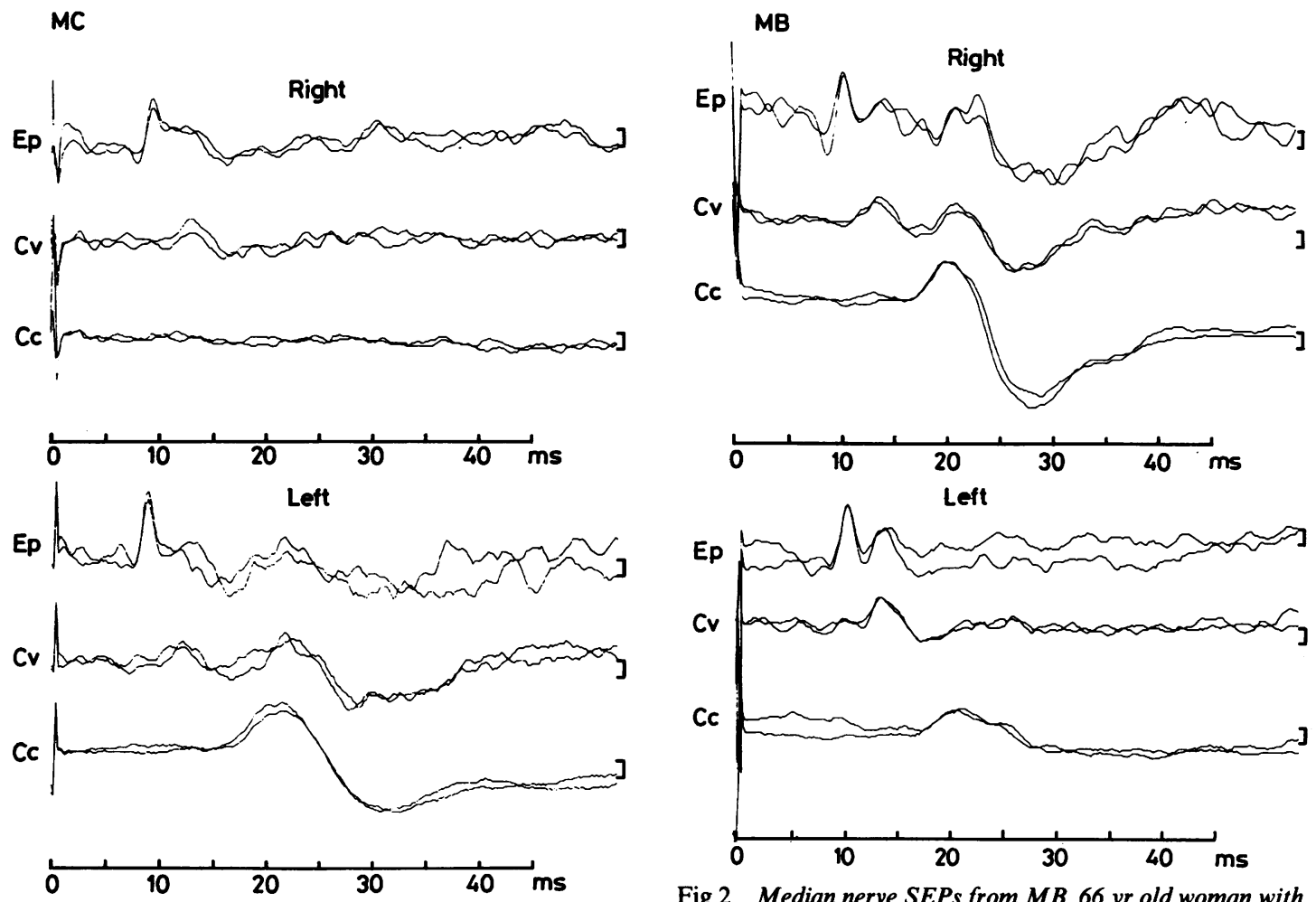

Fig 1 Median nerve SEPs from MC, 72 yr old woman with a left temporoparietal infarct. Recordings were made from electrodes at Erb's point (Ep), Second cervical vertebrae $(C v)$ and contralateral cortex $(C c)$. The trace shows an absent $\mathrm{N} 20$ and its following positivity from the appropriate side. The scale at end of each trace equals $2 \mu \mathrm{V}$.

indicate that delayed or low amplitude responses have a similar outcome to those with a normal SEP. It would be difficult to attribute the relevance of these findings to the site of the lesion as these numbers are too small. In the group with absent SEPs (eight patients) the outcome was variable depending on the site of the infarct. An absent SEP with cortical infarction had a poor outcome in the five cases (mean Barthel Index of 65) whereas with subcortical or brainstem infarction this correlated with a better outcome in the four cases (mean Barthel Index of 92). These numbers however are too small to draw statistical conclusions.

\section{Discussion}

According to the WHO classification of Impairments, Disabilities and Handicaps, ${ }^{20}$ a stroke will cause a specific neurological loss called an impairment with a resultant functional loss called a disability. In the

Fig 2 Median nerve SEPs from MB, 66 yr old woman with a right internal capsule infarct. Recordings were made from electrodes at Erb's point (Ep), Second cervical vertebrae $(\mathrm{Cv})$ and contralateral cortex $(\mathrm{Cc})$. The trace shows an absent positivity following a low amplitude N20 on left median nerve stimulation. The scale at end of each trace equals $2 \mu \mathrm{V}$.

rehabilitation of stroke patients, a functional loss (disability) is more important than neurological signs (impairment).

SEPs have been used previously in the assessment of stroke patients and their functional outcome however there was little distinction made between impairment and disability.

Liberson in $1966^{11}$ studied 15 aphasic patients. He concluded that severe dyphasia correlated with "suppression" of the SEP in eight out of 10 patients. Two had SEP "suppression" without severe dysphasia and one severe dysphasia without SEP "suppression". However, functional outcome was not considered.

Kusoffosky et al studied 16 hemiplegic patients and concluded that there was a good correlation between early SEPs and the subsequent development of motor activity especially in the upper limb. The small numbers made it difficult to draw statistical conclusions.

La Joie ${ }^{19}$ indicated a use in prognosis for mobility by using SEPs to determine proprioceptive loss in aphasic patients. Other studies ${ }^{2122}$ proposed that SEPs could 
be correlated with anosognosia using bilateral stimulation but no assessment of functional outcome was made.

Our study shows that there is a good correlation between SEP and functional outcome measured by length of stay and Barthel Index. The average length of stay was 29.56 days in those with normal SEPs, compared to 70.3 in those with abnormal SEPs. The Barthel Index was $\mathbf{9 5 . 9 3}$ in those with normal SEPs and 76.84 in those with abnormal SEPs. All of these results were significant at the 0.05 level.

Mortality figures obviously do not give a reflection of functional outcome but merely the disease state. Both length of stay and return home, have long been used as crude indirect measures of functional outcome. However, they both may be influenced by factors other than functional outcome such as family situations, other illnesses, availiblity of community resources and criteria for discharge. For populations within a certain community, these will tend to be evenly distributed, so that other influences can be measured. Therefore, as the measurement of functional outcome depends on multiple factors, a statistically significant number of patients are required and no single factor will reliably predict functional outcome in an individual.

Placement at home or in a nursing home was found not to be sensitive enough as a measure of outcome in our group of patients on statistically significant level. It tends to reflect extremely poor functional outcome whereas the majority of survivors of stroke nowadays go home.

Length of stay, although a coarse measure of outcome is easily understood and has direct practical significance. In different hospitals, different outpatient and inpatient services and policies will cause different lengths of stay for patients with similar disabilities.

The Barthel Index may differ between institutions and scorers depending on a number of variables. It has been found that measurement of specific activities in a clinic setting may not accurately reflect the ability to perform that task outside the clinic. There has been a gradual acceptance that measures of functional outcome can only be correct for groups of patients who have wide variations in performance of measured activities. Anecdotal experience in this circumstance is apt to be misleading as outcome may depend on a large number of variables that are not measured or are difficult to measure, such as social support.

Allowing for the difficulties with outcome measures, the conclusion that lengths of stay and functional outcome as measured by the Barthel Index correlate with somatosensory evoked potentials results on a statistically significant level is valid. By providing a practical, early and objective prediction of outcome, they can be used to assess the effectiveness of different treatments which minimise disability.

Preliminary findings suggest that the relationship of $\frac{Z}{D}$ SEP abnormality to the outcome measures may be dependent on the site of the infarct; an absent SEP response in a corticol infarction having a much poorer $\bar{O}$ outcome than a subcorticol lesion. However a larger $D$ study is required to confirm the relationship of the SEP, outcome and the site of the stroke. The size of the $\frac{}{\bar{y}}$. stroke itself will also have an effect on the outcome and $\stackrel{\text { \% }}{5}$ perhaps the SEP reflects this, but more patients and a :more detailed assessment of the CT would be required $\overrightarrow{\vec{F}}$ to draw any statistically significant conclusions. As allo but one patient had his stroke in the distribution of the $\frac{C}{0}$ middle cerebral artery after patients with normal CT $\frac{\bar{\sigma}}{\bar{s}}$ and brainstem strokes were excluded, no conclusions $\underset{\nabla}{\mathrm{Q}}$ can be drawn about the value of SEP in those patients with lesions in say the posterior circulation or anterior $\stackrel{\Phi}{s}$ cerebral artery territory.

It has been suggested that the SEP reflects conduc- $\overrightarrow{\vec{\omega}}$ tion in various sensory pathways. In our study, $68 \%$ of patients with abnormal SEPs had sensory loss and $81 \%$ of patients with normal SEPs had abnormal sensation. A closer association between SEP and in sensory pathways could not be determined in our i study as those with sensory loss usually had af in modalities affected to various degrees. Our stuक्ष $\vec{N}$ confirms other studies that have found a stroig association between SEP and sensory examination. $\unrhd$ -

Giblin $^{2}$ found that out of 42 stroke patients, SEPS T1 correlated with clinical abnormalities in only patients. Larson ${ }^{10}$ looked at six patients sequentiatity and found that clinical improvement correlated wi improvement in SEP in four, but in two patients the $\vec{\varphi}$ improved clinically but not in SEP. In 1970, Williamson et al $^{22}$ looked at 42 stroke patients. They excluded $\square$ severe dysphasics and in addition excluded three as technically unsatisfactory results because of lack of

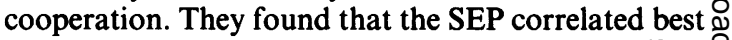
with sensory impairment. Despland and Regli ${ }^{15}$ in $\bar{\varnothing}$ 1985 investigated 70 patients with clearly defined $\stackrel{\circ}{\rightarrow}$ cortical or subcortical vascular lesions confined to the $\bar{O}$ post central gyrus. They used SEPs within one week of the CVA, and compared the site of the lesion with the clincal examination and SEP result. They found that a normal N20 component correlated with mild to moderate tactile or vibration deficit. The abolition of 0 N20 always correlated with severe lemniscal dysfunc- $\frac{5}{3}$ tion and astereognosis. Follow up after many months in 17 patients showed that the SEP may change. Functional outcome was again not looked at.

Since Van Buskirk and Webster in $1955^{23}$ described 윽 the prognostic value of sensory impairment in the $>$ functional outcome of hemiplegic stroke, many others have confirmed this, but is is rarely emphasised in No clinical practice or in the literature. It may be there is an overemphasis on motor recovery or that the $\mathcal{N}$ 
measurement of sensory deficit is not as obvious and is more subjective and less quantifiable than the motor deficit.

Our study confirms that sensory impairment is a statistically significant measure of functional outcome. SEPs can offer the advantages of subjectivity and quantification over standard sensory examination, and appear as accurate as sensory examination in predicting functional outcome. There is some indication that specific SEP abnormalities may be a better indicator of ultimate prognosis but more detailed examination of the SEP and larger numbers would be necessary to do confirm this.

Combining SEP abnormality and sensory disturbance shows an even better correlation with poor outcome than either factor alone, but only when brainstem and CT negative strokes are included. (Length of stay was 83.44 days and Barthel Index 68.46 at a 0.003 level of significance). However, combined normal SEP and normal sensation did not correlate significantly $(0.058)$ with a good outcome probably as a result of some patients with normal sensation having a poor outcome. This may be related to insufficient numbers.

In clinical practice, SEPs will continue to be used for diagnostic purposes, for example in brainstem strokes and pure sensory disturbances where other tests may be normal. Although SEPs will not replace the clinical examination they can provide a more objective measure of sensory pathways and are particularly useful in patients with dysphasia, inattention or a decreased level of consciousness. They also provide a statistically significant method of determining outcome as measured by length of stay and Barthel Index. It remains to be seen whether specific SEP abnormalities will be a better predictor of outcome than a good clinical examination and further studies are needed. Like other methods of determining outcome, they are more suitable for cohorts and groups of patients than individuals.

\section{References}

1 Allen CM. Predicting the outcome of acute stroke: a prognostic score. J Neurol Neurosurg Psychiatry 1984;47:475-80.

2 Hurwitz LJ, Adams GF. Rehabilitation of Hemiplegia: Indices of Assessment and Prognosis. Br Med J 1972;1:94-98.

3 Prescott RJ, Garraway WM, Akhtar AJ. Predicting Functional Outcome following Acute Stroke using a Standard Clinical
Examination. Stroke 1982;13:641-7.

4 Lehmann J, De Lateur BJ, Fowler RS, et al. Stroke Rehabilitation: Outcome and Prediction. Arch Phys Med Rehabil 1975;56: 383-9.

5 Greenberg RP, Ducker TB. Evoked Potentials in the clinical neurosciences. J Neurosurg 1981;56:1-18.

6 Mahoney FI, Barthel DW. Functional evaluation: The Barthel Index. Maryland State Medical Journal 1965;14:61-65.

7 Dawson GD. Cerebral responses to electrical stimulation of peripheral nerve in man. J Neurol Neurosurg Psychiatry 1947;10:134-40.

8 Chiappa KH. Evoked Potentials in Clinical Medicine. New York: Raven Press, 1983.

9 Yiannikas C, Shahani BT, Young RR. The investigation of traumatic lesions of the brachial plexus by electomyography and short latency somatosensory potentials evoked by stimulation of multiple peripheral nerves. J Neurol Neurosurg Psychiat 1983;46:1014-22.

10 Larson SJ, Sancer A, Baker JB. Evoked Cortical Potentials in Patients with Stroke. Circulation (Supplement II) 1966;33: 15-19.

11 Liberson W. Study of evoked potentials in aphasics. Am J Phys Med 1966;45:135-42.

12 Kusoffsky A, Wadon I, Nilsson B. The Relationships between sensory impairment and motor recovery in patients with hemiplegia. Scand J Rehab Med 1982;14:27-32.

13 Shibaski H, Yamashita Y, Tsuji S. Somatosensory Evoked Potentials: Diagnostic Criteria and Abnormalities in Cerebral Lesions. J Neurol Sci 1977;34:427-39.

14 Obeso JA, Marti-Masso JF, Carrera N. Somatosensory evoked potentials: abnormalities with focal brain lesions remote from the primary sensortimotor area. Electoencephalogy Clin Neurophysiol 1980;49:59 -65.

15 Despland PA, Regli F. Somatosensory evoked response changes in patients with unilateral vascular lesions. Evoked Potentials: Neurophysiological and clinical aspects. Morocutti C, Rizzo PA, eds. Amsterdam: Elsevier Science Publisher BV, 1985;57-67.

16 Rappaport M, Hall K, Hopkins K, et al. Evoked Brain Potentials and Disability in Brain Damaged Patients. Arch Phys Med Rehabil 1977;58:333-8.

17 Greenberg RP, Newton P, Hyatt MS, Narayan RK, Becker DP. Prognostic implications of early multimodality evoked potentials in severely head-injured patients. $J$ Neurosurg 1981;55: 227-36.

18 Cant BR, Hume AL, Judson JA, Shaw NA. The Assessment of Severe Head Injury by Short-Latency Somatosensory and Brain-Stem Auditory Evoked Potentials. Electoencephalogy Clin Neurophysiol 1986;65:188-95.

19 La Joie WJ, Reddy NM, Melvin JL. Somatosensory Evoked Potentials: Their Predictive Value in Right Hemiplegia. Arch Phys Med Rehabil 1982;63:223-6.

20 World Health Organization(WHO). International Classification of Impairments, Disabilities and Handicaps. Geneva: World Health Organization, 1980:184.

21 Giblin DR. Somatosensory evoked potentials in healthy subjects and in patients with lesions of the nervous system. Ann NY Acad Sci 1964;112:93-142.

22 Williamson PD, Goff WR, Allison T. Somatosensory evoked potentials in patients with unilateral cerebral lesions. Electoencephalogy Clin Neurophysiol 1970;28:566-75.

23 Van Buskirk C, Webster D. Prognostic Value of Sensory Deficit in Rehabilitation of Hemiplegics. Neurology 1955;5:407-11. 